VOL. II (1974), 77-88.

\title{
Regular completions of Cauchy spaces via function algebras
}

\section{R.J. Gazik and D.C. Kent}

\begin{abstract}
A regular completion with the universal property is obtained for each member of a certain class of Cauchy spaces by embedding the Cauchy space in a complete function algebra with the continuous convergence structure.
\end{abstract}

\section{Preliminaries}

A Cauchy space $(X, C)$ is a set $X$ equipped with a Cauchy structure $C$; that is, $C$ is a set of filters on $X$ (called "Cauchy filters") subject to the following conditions:

$\left(c_{1}\right)$ the ultrafilter $\dot{x}$ containing $\{x\}$ is in $\mathrm{C}$ for all $x$ in $X$;

$\left(c_{2}\right) \quad F \in C$ and $G \geq F$ implies $G \in C$;

$\left(c_{3}\right)$ if $F, G \in C$ and $F \vee G \neq 0$ (meaning each set in $F$ intersects each set in $G$ ), then $F \cap G \in C$.

A Cauchy structure $C$ generates a convergence structure on $X$ defined as follows: $F \rightarrow x$ in $X$ if $\dot{x} \cap F \in C$. Keller [5] showed that a set of filters on $X$ satisfying $\left(c_{1}\right),\left(c_{2}\right)$, and $\left(c_{3}\right)$ is precisely the set of Cauchy filters for some uniform convergence structure (see [3]) on $X$. A definitive study of Cauchy spaces has been made by Ramaley and Wyler ([8], [9]).

A Cauchy space $(X, C)$ is complete if each Cauchy filter is convergent. Each convergence space which admits a uniform convergence

Received 5 April 1974. 
structure (see [5] for a characterization of such spaces) can be regarded as a complete Cauchy space if the Cauchy structure is taken to be the set of all Cauchy filters. A function $f:(X, C) \rightarrow\left(X_{1}, C_{1}\right)$ is cauchycontinuous if $F \in C$ implies $f(F) \in C_{1}$. The pair $\left(\left(x_{1}, C_{1}\right), j\right)$ is a completion of a Cauchy space $(X, C)$ if $\left(X_{1}, C_{1}\right)$ is a complete Cauchy space, and $j:(X, C) \rightarrow\left(X_{1}, C_{1}\right)$ is a dense Cauchy embedding. If, in addition, $F \rightarrow x$ in $X$ implies the existence of a filter $G \in C$ such that $j G+x$ in $X$ and $F \geq \operatorname{cl}_{X}(j G)$, the completion is said to be strict.

Reed $[10]$, showed that each completion of a Cauchy space $(X, C)$ induces a completion of each uniform convergence space $(X, J)$ for which $C$ is the set of J-Cauchy filters. A Cauchy space $(X, C)$ is regular if $c l_{X} F \in C$ whenever $F \in C$. It is shown in [6] that a strict, regular, Hausdorff completion of a Cauchy space, if it exists, is unique up to Cauchy-homeomorphism.

The Cauchy filters in a Cauchy space $(X, C)$ are partitioned into Cauchy equivalence classes by the following equivalence relation: $F \sim G$ if and only if $F \cap G \in C$. Reed [10] used the set $\{[F]: F \in C\}$ of these equivalence classes to construct a family of completions for any Cauchy space. In particular, she showed that every Hausdorff Cauchy space (that is, Hausdorff in its underlying convergence structure) has a strict Hausdorff completion; we will make use of this fact later.

A Cauchy space $(X, C)$ is said to be pseudo-topological if a filter $F$ is in $C$ whenever all ultrafilters finer than $F$ are in the same Cauchy equivalence class relative to $C$. A convergence space is pseudotopological if a filter converges to $x$ whenever each refining ultrafilter converges to $x$. Note that a pseudo-topological convergence space can be regarded as a complete pseudo-topological Cauchy space.

Let $X$ be a convergence space, $C(X)$ the $R$-algebra of all continuous real valued functions on $X$. The continuous convergence structure $c$ on $X$ is the coarsest relative to which the function $w: C(X) \times X \rightarrow R$, defined by $w(f, x)=f(x)$, is continuous. The resulting convergence function space $C_{c}(X)$ has been studied extensively 
(see, for instance, [1], [2], [4], [11], and [12]). The natural map $i: X+C_{c} C_{c}(X)$ is defined by $i(x)=F_{x}$, where $F_{x}(f)=f(x)$, all $f \in C(X)$. Binz [1], has shown that $i(X)=\operatorname{hom} C_{c}(X)$, the set of all continuous algebra homomorphisms from $C_{c}(X)$ into $R$ which preserve the multiplicative identity. The space $X$ is said to be c-embedded if the function $i$ is an embedding. In order to state a characterization of c-embedded spaces, we need a few preliminary definitions.

Starting with a space $X$, let $\omega X$ denote the finest completely regular topological space coarser than $X$ on the same underlying set; $\omega X$ is called the completely regular modification of $X$. The fact that $C(X)=C(\omega X)$ is useful and will be used without explicit mention in what follows. We will use the symbol "cl $X$ " to represent the closure operator for any space $X, X$ is said to be w-regular if ${ }^{c 1}{ }_{\omega X} F+x$ in $X$ whenever $F \rightarrow x$ in $X$. The first proposition is established in [12] and, implicitly, in [11].

PROPOSITION 1.1. A space $X$ is c-embedded iff $X$ is pseudotopological, Hausdorff, and w-regular.

Binz [2], has shown that the space $C_{c}(X)$ is always c-embedded.

Next, let $(X, C)$ be a Cauchy space, and let $C^{\wedge}(X, C)$ be the $R$-algebra of all real-valued Cauchy-continuous functions on $(X, C)$. Let $C^{\wedge}$ denote the continuous Cauchy structure on $C^{\wedge}(X, C)$, defined as follows: a filter $\Phi$ on $C^{\wedge}(X, C)$ is in $C^{\wedge}$ iff $\Phi(F)$ is $R$-Cauchy (that is, $R$-convergent) whenever $F \in C$. If one defines the notion of product Cauchy structure in the natural way, then there is no difficulty in establishing the following proposition; we omit the details.

PROPOSITION 1.2. For any Cauchy space $(X, C), \mathrm{C}^{\wedge}$ is the coarsest Cauchy structure on $C^{\wedge}(X, C)$ relative to which the function $w: C^{\wedge}(X, C) \times(X, C) \rightarrow R$, defined by $w(f, x)=f(x)$, is Cauchycontinuous.

PROPOSITION 1.3. For any Cauchy space $(X, C)$, the Cauchy function space $\left(C^{\wedge}(X, C), C^{\wedge}\right)$ is complete.

Proof. Let $\Phi \in \mathrm{C}^{\wedge}$, and let $f: X \rightarrow R$ be defined by 
$f(x)=\lim \Phi(\dot{x})$, all $x \in X$.

To show that $f \in C^{\wedge}(X)$, let $F \in C$; then $\Phi(F) \rightarrow a$, for some $a \in R$. Let $W$ be a closed neighborhood of $a$ in $R$; then there is $A \in \Phi$ and $F \in F$ such that $A(F) \subset W$. If $x \in F$, then $A(x) \subset W$, and so $f(x) \in \operatorname{cl}_{R} A(x) \subset W$. Thus $f(F) \subset W$, and $f(F) \rightarrow a$ in $R$.

To show that $\Phi \rightarrow f$, it must be shown that $\Phi \cap \dot{f}^{\circ} \in C^{\wedge}$. If $F \subset X$, $A \in \Phi$, and $x \in F$, then $A(F) \in \Phi(\dot{x})$, and so $f(F) \subset \mathrm{cl}_{R} A(F)$. Thus, if $F \in C, \dot{f}(F)>c]_{F} \Phi(F)$, and hence $\dot{f}(F)$ and $\Phi(F)$ must converge to the same point in $R$.

A complete Cauchy space is merely a convergence space in which the convergent filters are designated to be Cauchy. Thus the Cauchy structure $C^{\wedge}$ on the function space is somewhat redundant and will usually be deleted. Also, it is convenient to shorten the notation from $C^{\wedge}(X, C)$ to $C^{\wedge}(X)$ if there is no danger of confusing which Cauchy structure on $X$ is being considered.

The next proposition follows by inspection of the respective definitions .

PROPOSITION 1.4. If $(X, C)$ is a complete Cauchy space, then $C^{\wedge}(X)$ coincides with $C_{c}(X)$.

Reed [10], proved that each Hausdorff Cauchy space $(X, C)$ has a strict, Hausdorff completion $\left(\left(x_{1}, C_{1}\right), j\right)$ with the regular extension property. The latter term means that each Cauchy-continuous function from $(X, C)$ into a Hausdorff, regular, complete Cauchy space $\left(x_{2}, C_{2}\right)$ has a Cauchy-continuous extension $f_{1}:\left(x_{1}, C_{1}\right) \rightarrow\left(x_{2}, C_{2}\right)$. The existence of such a completion (but not the details of the construction) are needed for the following proposition.

PROPOSITION 1.5. Let $(X, C)$ be a Hausdorff Cauchy space, and let $\left(\left(X_{1}, C_{1}\right), j\right)$ be a strict, Hausdorff completion with the regular extension property. Then $C^{\wedge}(X)$ and $C^{\wedge}\left(X_{1}\right)$ are both isomorphic and homeomorphic.

Proof. Let $\Psi: C^{\wedge}(X) \rightarrow C^{\wedge}\left(X_{1}\right)$ be defined by $\Psi(f)=f_{1}$, where $f_{1}$ 
is the extension from $X$ to $X_{1}$ whose existence is derived from the regular extension property. The uniqueness of $f_{1}$ is guaranteed by the Hausdorff property, and so $\Psi$ is well defined. It is a trivial matter to verify that $\Psi$ is an algebra isomorphism.

To show that $\Psi$ is Cauchy continuous, let $\Phi$ be $C^{\wedge}(X)$-Cauchy and let $A \in C_{1}$. Since $X_{1}$ is complete, $A$ converges in $X_{1}$, and by the strictness of the completion, there is $F \in C$ such that $A \geq c{ }_{X_{1}} j F$. It then follows easily that $(\Psi \Psi)(A) \geq \mathrm{cl}_{R} \Phi(F)$. But $\Phi(F)$ is $R$-convergent, by definition of the Cauchy structure on $C^{\wedge}(X)$, and, since $R$ is regular, $(\Psi \Phi)(A)$ is likewise $R$-convergent. Thus $\Psi \Phi$ is $C^{\wedge}\left(X_{1}\right)$-Cauchy.

To show that $\Psi^{-1}$ is continuous, let $\Lambda$ be a $C^{\wedge}\left(X_{1}\right)$-Cauchy filter, and let $F \in C$. Then $\left(\Psi^{-1} \Lambda\right)(F) \geq(j F)$, and it follows that $\Psi^{-1} \Lambda$ is $C^{\wedge}(X)$-Cauchy.

COROLLARY 1.6. For any Hausdorff Cauchy space $(X, C)$, there is a convergence space $X_{1}$ such that $C^{\wedge}(X)$ is isomorphic and homeomorphic to $c_{c}\left(X_{1}\right)$. Consequently, $C^{\wedge}(X)$ is a Hausdorff, w-regular, pseudotopological convergence space.

Proof. These statements follow immediately from Proposition 1.1, the remark following Proposittion 1.1, Proposition 1.4, and Proposition 1.5.

It follows from the preceding results that, for any Cauchy space $(X, C), C^{\wedge} C^{\wedge}(X)$ is equal to $C_{c^{\wedge}} C^{\wedge}(X)$ and is equivalent, in the sense of Corollary 1.6, to $C_{c} C_{c}\left(X_{1}\right)$, where $\left(X_{1}, C_{1}\right)$ is the completion of

Proposition 1.5. A Cauchy space $(X, C)$ is said to be $c^{\wedge}$-embedded if the function $i:(X, C) \rightarrow C^{\wedge} C^{\wedge}(X)$, where $i(x)(f)=f(x)$ for all $f$ in $C^{\wedge}(X)$, is a Cauchy embedding. In order to obtain an internal characterization of $c^{\wedge}$-embedded spaces, we will introduce several new Cauchy space concepts.

For any Cauchy space $(X, C)$, let $\mu X$ be the set $X$ with the weak topology induced by the set of functions $C^{\wedge}(X)$. Since $C^{\wedge}(X) \subset C(X)$, it follows that $\mu X \leq \omega X$, and $\mu X=\omega X$ if $(X, C)$ is complete. $(X, C)$ is 
said to be $\mu$-regular if ${ }^{\mathrm{cl}}{ }_{\mu X} F \in C$ whenever $F \in C$. It is clear that $\mu$-regular implies $\omega$-regular implies regular.

A Cauchy space $(X, C)$ is said to be Cauchy-separated if $X$ is Hausdorff and, whenever $F, G \in C$ and $F \cap G \notin C$, there is $f \in C^{\wedge}(X)$ such that $\lim f(F) \neq \lim f(G)$ in $R$. In other words, $(X, C)$ is Cauchyseparated iff there are enough functions in $C^{\wedge}(X)$ to separate Cauchy equivalence classes. Since distinct points in a Hausdorff Cauchy space correspond to distinct equivalence classes, it follows that $\mu X$ is Hausdorff whenever $(X, C)$ is Cauchy-separated.

PROPOSITION 1.7. Let $(X, C)$ be a Cauchy space, $A$ a $\mu X$-closed subset of $X$, and $x \in X-A$. Then there is a function $f$ in $C^{\wedge}(X)$ such that $f(x)=0$ and $f(A)=1$.

Proof. Let $J$ be the uniformity on $X$ generated by sets of the form $J(\varepsilon, f)=\{(x, y):|f(x)-f(y)|<\varepsilon\}$, where $\varepsilon>0$ and $f \in C^{\wedge}(X, C)$. Note that the J-Cauchy filters are precisely those filters $F$ such that $f \in C^{\wedge}(X, C)$ implies that $f(F)$ is Cauchy on $R$. Thus, if $C_{J}$ is the set of J-Cauchy filters, $C \subset C_{J}$. It also follows from this construction that $C_{J}$ is an admissible Cauchy structure for $\mu X$.

Let $x$ and $A$ be as stated in the proposition, and let $\left(X_{1}, J_{1}\right)$ be a complete uniform space containing $(X, J)$. Then there is an $X_{1}$-closed set $B \subset X_{1}$ such that $A=X_{1} \cap B$. Since $X_{1}$ is a completely regular topological space, there is $g \in C\left(X_{1}\right)$ such that $g(x)=0$ and $g(B)=1$. If $f$ is the restriction of $g$ to $X$, then $f \in C^{\wedge}\left(X, c_{J}\right), f(x)=0$, and $f(A)=1$. Since $C \subset C_{J}, C^{\wedge}\left(X, C_{J}\right) \subset C^{\wedge}(X, C)$, and the proof is complete.

PROPOSITION 1.8. A Cauchy space $(X, C)$ which has an w-regular, Hausdorff completion is $\mu$-regular and Cauchy-separated.

Proof. Let $\left(\left(X_{1}, C_{1}\right), j\right)$ be a Hausdorff, w-regular completion of $(X, C)$. We will first show that $(X, C)$ is $\mu$-regular. Let $F \in C$; then $\operatorname{cl}_{\omega X_{1}}(j F) \in C_{1}$. If. $A \subset X$, then 


$$
{ }^{c l}{ }_{1}(j A)=n\left\{f^{-1}(B): B \text { closed subset of } R, j A \subset f^{-1}(B), f \in C\left(X_{1}\right)\right\},
$$

by Proposition 1.7 , and

$$
\mathrm{cl}_{\mu X} A=\cap\left\{f^{-1}(B): B \text { closed subset of } R, A \subset f^{-1}(B), f \in C^{\wedge}(X)\right\} .
$$

If $f \in C\left(X_{1}\right)$, then the restriction of $f$ to $X$ is in $C^{\wedge}(X)$. Thus $j\left(\mathrm{cl}_{\mu X} A\right) \subset \mathrm{cl}_{\omega X_{1}}(j A)$, and it follows that $\mathrm{cl}_{\mu X} F \geq j^{-1}\left(\mathrm{cl}{ }_{\omega X}(j F)\right)$. Thus ${ }^{c l}{ }_{\mu X} F \in C$, and $(X, C)$ is $\mu$-regular.

To show that $(X, C)$ is Cauchy separated, let $F, G \in C$ and $F \cap G \vDash C$. Then $j F$ and $j G$ are in distinct Cauchy equivalence classes in $C_{1}$, and so $j F \rightarrow a$ in $X_{1}, j G \rightarrow b$ in $X_{1}$, and $a \neq b$. Since $X_{1}$ is w-Hausdorff, there is a function $f$ in $C\left(X_{1}\right)$ which separates the points $a$ and $b$. If $g=f \circ j$, then $g \in C^{\wedge}(X)$, and $\lim g F \neq \lim g G$.

\section{The natural completion}

In this section, we will characterize the $\hat{c}$-embedded Cauchy spaces both internally and externally; the external characterization leads to what we call the natural completion. The natural completion is an $w$-regular, Hausdorff, pseudo-topological completion obtained by embedding $(X, C)$ into $C^{\wedge} C^{\wedge}(X)$ under the natural injection $i$.

Let $\operatorname{hom}^{\wedge} C^{\wedge}(X)$ denote the subspace of $C^{\wedge} C^{\wedge}(X)$ consisting of all continuous algebra homomorphisms from $C^{\wedge}(X)$ into $R$ which preserve the multiplicative identity. $\operatorname{hom}^{\wedge} C^{\wedge}(X)$ coincides with $\operatorname{hom}_{C} C^{\wedge}(X)$, and by Corollary 1.6, $\operatorname{hom}^{\wedge} C^{\wedge}(X)$ is an w-regular, Hausdorff, pseudo-topological convergence space, since all of these properties are inherited from ${ }_{C} C^{\wedge}(X)$. We will assume that $\operatorname{hom}^{\wedge} C^{\wedge}(X)$ is equipped with the Cauchy structure inherited from $C^{\wedge} C^{\wedge}(X)$.

PROPOSITION 2.1. Let $(X, C)$ be a Hausdorff Cauchy space, $i:(X, C) \rightarrow C^{\wedge} C^{\wedge}(X)$ the natural function.

(a) $i(X)$ is a dense subset of $\operatorname{hom}^{\wedge} C^{\wedge}(X)$.

(b) $i$ is Cauchy-continuous. 
(c) If $(X, C)$ is Cauchy-separated, then i is one-to-one.

Proof. Let $\left(\left(X_{1}, C_{1}\right), j\right)$ be a strict, Hausdorff completion of $(X, C)$ (see remarks preceding Proposition 1.5). Consider the following commutative diagram:

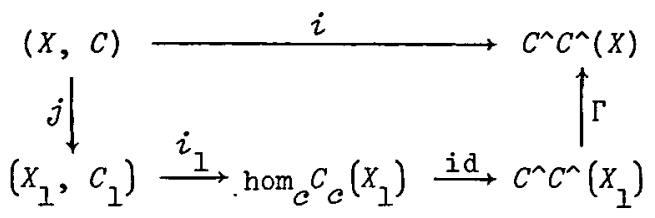

In this diagram, id is the identity map and $\Gamma$ the homeomorphism and algebra isomorphism derived from the homeomorphism $\Psi: C^{\wedge}(X) \rightarrow C^{\wedge}\left(X_{1}\right)$ of Proposition 1.5. By the work of Binz [1], $i_{1}$ is always continuous and onto hom $C_{c} C_{1}\left(X_{1}\right)$. Thus $i$ is a composition of Cauchy-continuous functions (recall that all the spaces in the above diagram except $(X, C)$ are complete Cauchy spaces, and continuous functions between complete Cauchy spaces are Cauchy continuous). $j X$ is dense in $X_{1}$, and therefore $i_{1} j X$ is dense in $\operatorname{hom}_{c} C_{c}\left(X_{1}\right)$. Since $\Gamma$ is an algebra isomorphism, $\Gamma$ carries $\operatorname{hom}_{c} C_{c}\left(X_{1}\right)$ onto $\operatorname{hom}^{\wedge} C^{\wedge}(X)$, and $(a)$ is thus established.

Finally, assume that $(X, C)$ is Cauchy-separated, and let $i(x)=i(y)$. This implies $f(x)=f(y)$, all $f \in C^{\wedge}(X)$. But $\mu X$ has the weak topology induced by $C^{\wedge}(X)$, and $x=y$ follows from the fact that $\mu X$ is Hausdorff whenever $(X, C)$ is Cauchy-separated (see the remark preceding Proposition 1.7).

Let $(X, C)$ be a Cauchy space. A collection $B$ of subsets of $X$ will be called a Cauchy covering system if each member of $C$ contains a member of $B$. This definition is the obvious extension to Cauchy spaces of the notion of "covering system" defined for convergence spaces in [4].

Starting with a Cauchy system $B$ for $(X, C)$, let $U$ be the uniformity on the set $C^{\wedge}(X)$ with basic sets of the form $T\left(B_{1}, \ldots, B_{n}, \varepsilon\right)=\left\{(f, g):|f(x)-g(x)|<\varepsilon\right.$ for all $\left.x, y \in \cup B_{i}\right\} \cup \Delta$, where $\Delta$ is the diagonal in $C^{\wedge}(X) \times C^{\wedge}(X),\left\{B_{1}, \ldots, B_{n}\right\}$ is an arbitrary finite subcollection of $B$, and $\varepsilon>0$. Let $\overline{0}$ be the zero 
function in $C^{\wedge}(X)$, and $N(\overline{0})$ the neighborhood filter at $\overline{0}$ in the uniform topology on $C^{\wedge}(X)$.

LEMMA 2.2. In the notation of the preceding paragraph, $N(\overline{0}) \in \mathrm{C}^{\wedge}$, the continuous Cauchy structure on $C^{\wedge}(X)$.

Proof. Let $H \in C$; then $H$ contains a set $B \in B$, and, for any $\varepsilon>0,(T(B, \varepsilon)(\overline{0}))(B) \subset(-\varepsilon, \varepsilon)$.

THEOREM 2.3. The following statements about a Cauchy space $(X, C)$ are equivalent:

(a) $(X, C)$ is $c^{\wedge}$-embedded;

(b) $\left(\operatorname{hom}^{\wedge} C^{\wedge}(X), i\right)$ is a completion of $(X, C)$;

(c) $(X, C)$ has an w-regular, Hausdorff, pseudo-topological completion;

(d) (X, C) is u-regular, Cauchy-separated, and pseudotopological.

Proof. (a) implies (b). By Proposition 2.1, $i(X)$ is a dense subset of $\operatorname{hom}^{\wedge} C^{\wedge}(X)$. hom ${ }^{\wedge} C^{\wedge}(X)$ is a closed subspace of the complete Cauchy space $C^{\wedge} C^{\wedge}(X)$, so $\operatorname{hom}^{\wedge} C^{\wedge}(X)$ is also a complete Cauchy space.

(b) implies (c). We noted in the second paragraph of Section 2 that hom^ $C^{\wedge}(X)$ is $\omega$-regular, Hausdorff, and pseudo-topological.

(c) implies (d). See Proposition 1.8.

(d) implies $(a)$. The function $i:(X, C) \rightarrow \operatorname{hom}^{\wedge} C^{\wedge}(X)$ is Cauchycontinuous and one-to-one by Proposition 2.1. Thus it remains only to show that if $G$ is a filter on $X$ such that $i G$ is convergent in $\operatorname{hom}^{\wedge} C^{\wedge}(X)$, then $G \in C$. Since $(X, C)$ is pseudo-topological, it is sufficient to show:

(1) each ultrafilter finer than $G$ is in $C$;

(2) all ultrafilters finer than $G$ are in the same equivalence class.

To establish (1), assume that $F$ is an ultrafilter, $F \geq G$, and $F \notin C$. Then $i(F)$ converges in hom^ $C^{\wedge}(X)$. Since $F \vDash C$, and $(X, C)$ is $\mu$-regular, $F \neq c{ }_{\mu X} X^{H}$ for all $H \in C$. Thus, for each $H \in C$, there 
is $H_{H}$ in $H$, but not in $F$, such that $H_{H}$ is $\mu X$-closed. The set $B=\left\{H_{H}: H \in C\right\}$ is a Cauchy covering system for $(X, C)$. Let $U$ be the uniformity on the set $C^{\wedge}(X)$ derived from $B$, as in the paragraph preceding Lemma 2.2, and let $N(\overline{0})$ be the uniform neighborhood filter at the constant function $\overline{0}$. By Lemma $2,2, N(\overline{0}) \in C^{\wedge}$. Since $i(F)$ is hom ${ }^{\wedge} C^{\wedge}(X)$-Cauchy, $i(F)(N(\overline{0})) \rightarrow 0$; which implies that $(N(\overline{0}))(F) \rightarrow 0$ in $R$.

Let $W$ be a neighborhood of 0 in $R$ not containing 1 . Then there are sets $B_{1}, \ldots, B_{n}$ in $B, \varepsilon>0$, and $F \in F$ such that $\left(T\left(B_{1}, \ldots, B_{n}, \varepsilon\right)\right)(F) \subset W$. Since $F$ is an ultrafilter and $B_{i} k F$ for $i=1, \ldots, n, F$ is not a subset of $B_{0}=U\left\{B_{i}: i=1, \ldots, n\right\}$. Let $x \in F-B_{0}$. Since $B_{0}$ is $\mu X$-closed, it follows from Proposition 1.7 that there is a function $f \in C^{\wedge}(X)$ such that $f(x)=1$ and $f\left(B_{0}\right)=0$. By this construction, $f \in T\left(B_{1}, \ldots, B_{n}, \varepsilon\right)$, but $f(x) \vDash W$ for $x \in F$, a contradiction. This contradiction establishes that $F \in C$, and assertion (I) is proved.

To establish (2), suppose that $F$ and $H$ are ultrafilters finer than $G$; thus $i(F \cap H) \geq i(G)$. Let $f \in C^{\wedge}(x)$. Then $(i(F \cap H))(\dot{f})$ is equal to $f(F) \cap f(H)$, and so the latter filter is Cauchy in $R$. Thus $f(F)$ and $f(H)$ converge to the same point in $R$, and this is true for all $f \in C^{\wedge}(X)$. Thus, since $(X, C)$ is Cauchy-separated, $F \cap G \in C$. This completes the proof of Theorem 2.3.

If $(X, C)$ is a $c^{\wedge}$-embedded Cauchy space, we will refer to hom^ $C^{\wedge}(X)$ as the natural completion of $(X, C)$. As our concluding result in this paper, we will prove that the natural completion of a $c^{\wedge}$-embedded space has the universal property in the class of all $c^{\wedge}$-embedded Cauchy spaces.

Let $\phi:(X, C) \rightarrow\left(X_{1}, C_{1}\right)$ be a Cauchy continuous function from one $c^{\wedge}$-embedded Cauchy space into another. Then it is a routine matter to show that the function $\phi_{1}: C^{\wedge}\left(X_{1}\right) \rightarrow C^{\wedge}(X)$, defined by $\phi_{1}(f)=f \phi$ for all $f \in C^{\wedge}\left(x_{1}\right)$, is a Cauchy-continuous algebra homomorphism. 
THEOREM 2.4. If $(X, C)$ is a $c^{\wedge}$-embedded Cauchy space, $\left(X_{1}, C_{1}\right)$ a complete $c^{\wedge}$-embedded Cauchy space, and $\phi:(X, C) \rightarrow\left(X_{1}, C_{1}\right)$ is Cauchy-continuous, then $\phi$ has a unique Cauchy-continuous extension $\psi: \operatorname{hom}^{\wedge} C^{\wedge}(X) \rightarrow\left(X_{1}, C_{1}\right)$.

Proof. Define the natural function $\phi_{2}: C^{\wedge} C^{\wedge}(X) \rightarrow C^{\wedge} C^{\wedge}\left(X_{1}\right)$ in the manner described in the preceding paragraph. Let $\sigma$ be the restriction of $\phi_{2}$ to $\operatorname{hom}^{\wedge} C^{\wedge}(X)$. Since $\phi_{2}$ is a Cauchy-continuous homomorphism, $\sigma: \operatorname{hom}^{\wedge} C^{\wedge}(X) \rightarrow \operatorname{hom}{ }^{\wedge} C^{\wedge}\left(X_{1}\right)$ is Cauchy-continuous. Since $\left(X_{1}, C_{1}\right)$ is complete, $\left(X_{1}, C_{1}\right)$ is Cauchy-homeomorphic to $\operatorname{hom}^{\wedge} C^{\wedge}\left(X_{1}\right)$. Let $\psi=i_{1}^{-1} \sigma$, where $i_{1}$ is the natural embedding from $X_{1}$ onto $\operatorname{hom}{ }^{\wedge}\left(X_{1}\right)$. It is clear from this construction that $\psi$ is an extension of $\phi$ (in the sense that $\phi=\psi i$ ) and also that this extension is unique.

\section{References}

[1] E. Binz, "Bemerkungen zu limitierten Funktionenalgebren", Math. Ann. 175 (1968), 169-184.

[2] E. Binz, "Zu den Beziehungen zwischen c-einbettbaren Limesräumen und ihren limitierten Funktionenalgebren", Math. Ann. 181 (1969), $45-52$.

[3] C.H. Cook and H.R. Fischer, "Uniform convergence structures", Math. Ann. 173 (1967), 290-306.

[4] William A. Feldman, "Axioms of countability and the algebra $C(X)$ ", Pacific J. Math. 47 (1973), 81-89.

[5] H.H. Keller, "Die Limes-Uniformisierbarkeit der Limesräume", Math. Ann. 176 (1968), 334-341.

[6] D.C. Kent and G.D. Richardson; "Regular completion of Cauchy spaces", Pacific J. Math. (to appear).

[7] Kelly McKennon, "The strict topology and the Cauchy structure of the spectrum of a $C^{*}$-algebra", submitted. 
[8] J.F. Ramaley and Oswald Wyler, "Cauchy spaces. I. Structure and uniformization theorems", Math. Ann. 187 (1970), 175-186.

[9] J.F. Ramaley and Oswald Wyler, "Cauchy spaces. II. Regular completions and compactifications", Math. Ann. 187 (1970), 187-199.

[10] Ellen E. Reed, "Completions of uniform convergence spaces", Math. Ann. 194 (1971), 83-108.

[11] M. Schroder, "Solid convergence spaces", Bull. Austral. Math. Soc. 8 (1973), 443-459.

[12] M. Schroder, G. Richardson, K. McKennon, D. Kent, "Continuous convergence in $C(X)$ ", Pacific J. Math. (to appear).

Department of Mathematics,

Arkansas State University,

State University,

Arkansas,

USA;

Department of Pure and Applied Mathematics, Washington State University,

Puliman, Washington, USA. 